Session 5: The connection between binary evolution and $\mathrm{PN}$ phenomena

Chair: Roberto Mendez 


\title{
Circumstellar spirals/shells/arcs: the message from binary stars
}

\author{
Hyosun Kim $\dagger$ \\ Academia Sinica Institute of Astronomy and Astrophysics, P.O. Box 23-141, Taipei 10617, \\ Taiwan (email: hkim@asiaa.sinica.edu.tw)
}

\begin{abstract}
A consensus has grown in the past few decades that binarity is key to understanding the morphological diversities of the circumstellar envelopes (CSEs) surrounding stars in the Asymptotic Giant Branch (AGB) to Planetary Nebula (PN) phase. The possible roles of binaries in their shaping have, however, yet to be confirmed. Meanwhile, recurrent patterns are often found in the CSEs of AGB stars and the outer halos of PNe, providing a fossil record of the mass loss during the AGB phase. In this regard, recent molecular line observations using interferometric facilities have revealed the spatio-kinematics of such patterns. Numerical simulations of binary interactions producing spiral-shells have been extensively developed, revealing new probes for extracting the stellar and orbital properties from these patterns. I review recent theoretical and observational investigations on the circumstellar spiral-shell patterns and discuss their implications in linking binary properties to the asymmetric ejection events in the post-AGB phase.
\end{abstract}

Keywords. stars: AGB and post-AGB, binaries: general, circumstellar matter, stars: evolution, stars: fundamental parameters, stars: imaging, stars: late-type, stars: mass loss, stars: winds, outflows, submillimeter

\section{Introduction}

Since the launch of the Hubble Space Telescope (HST) in 1990, there have been many unexpected discoveries. One major finding among those is the recurrent patterns in the CSEs of sources in late stellar evolutionary phases (see Fig. 1), with AFGL 2688 being the archetypical target (Sahai et al. 1998). The HST image revealed its nearly concentric rings, which are interpreted as the limb-brightened surfaces of probable spherical shelllike structures. The pattern appear disconnected near the equatorial plane and brightest along the polar axis of the nebula due to the nonuniform illumination by direct starlight.

$\mathrm{Su}$ (2004) first summarized the properties of 10 sources showing the ring/arc patterns. Corradi et al. (2004) and Ramos-Larios et al. (2016) searched for the faint patterns in a systematic way by processing the HST and Spitzer archival imaging data, increasing the accumulated number of objects possessing the recurrent patterns to $\sim 60$. Recent molecular line observations using e.g., the Atacama Large Millimeter/submillimeter Array (ALMA) and Karl G. Jansky Very Large Array (JVLA) have been discovering the previously unknown spirals and shells. Table 1 shows some notable works in observations and theories on the recurrent patterns.

The kinematic timescales of the pattern intervals $\left(10^{2}-10^{3}\right.$ years $)$ are inconsistent with either stellar pulsation or thermal pulsation. Su (2004) showed that the timescales show no distinguishable trend along the evolutionary phase, implying that the ring/arc patterns are not a transient phenomenon. This is in conflict with one-dimensional timedependent hydrodynamics calculations with ionization taken into account, resulting in 

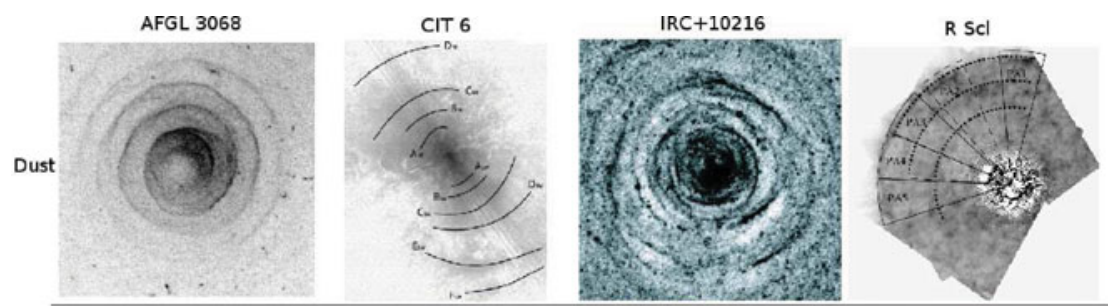

Mira
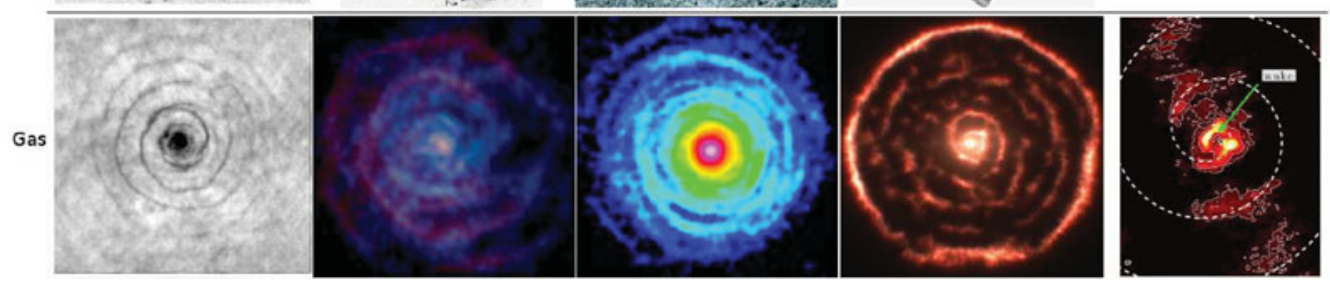

Figure 1. Upper panels: dust scattered light images of AFGL 3068 (Morris et al. in prep.), CIT 6 (Schmidt et al. 2002), IRC+10216 (Leão et al. 2006), and R Scl (Olofsson et al. 2010). Lower panels: CO center-frequency images of AFGL 3068 (Kim et al. 2017), CIT 6 (Kim et al. 2015b), IRC+10216 (Cernicharo et al. 2015), R Scl (Maercker et al. 2012), and Mira (Ramstedt et al. 2014). The sets of upper and lower panels are scaled in size.

\begin{tabular}{|c|c|}
\hline 1994 & Soker - spiral due to wide binary motion \\
\hline 1997 & Harpaz et al. - tidel interaction at periastron. \\
\hline 1998 & Sahai (AFGL 2688) $\quad \ldots$ and many more from HST \\
\hline 1999 & Mastrodemos \& Morris - binary \\
\hline 2004 & Corral et al. (search for faint patterns) \\
\hline 2006 & Mauron \& Huggins (AFGL 3068 spiral, HST) \\
\hline 2011 & Claussen (CIT6 rings, VLA) \\
\hline 2012 & $\begin{array}{l}\text { Kim \& Taam (a, b=parameter study, c=AFGL3068 spiral) } \\
\text { Maercker et al. (R Scl spiral) }\end{array}$ \\
\hline 2013 & Kim et al. (CIT6 spiral, VLA) \\
\hline 2014 & $\begin{array}{l}\text { Ramstedt et al. (Mira, ALMA) } \\
\qquad \text { Cernicharo et al. (IRC+10216 rose-window, IRAM) }\end{array}$ \\
\hline 2015 & Kim et al. (CIT6 spiral, SMA) \\
\hline 2016 & Kim et al. (AFGL 3068 spiral, ALMA) \\
\hline
\end{tabular}

Table 1. Research highlights on the concentric ring-/arc-like patterns in the circumstellar envelopes of AGB stars and pPNe/PNe. In 1990s and 2000s, the HST observations and theories were rather independently investigated. In the current decade, thanks to the operations of high-sensitivity millimeter/submillimeter telescopes (e.g., ALMA, JVLA), the new detection of such patterns and their theoretical understanding have led to significant and synergistic progress.

the patterns disappearing within $\sim 3000$ years after ionization starts (Meijerink et al. 2003). With the relevant model development (see §2) and the timescales consistent with the orbital periods of wide binaries, it is now commonly accepted that binary companions are responsible for modulating the mass distribution of CSEs in the recurrent form.

A remaining puzzle is their coexistence with bipolar structures in many pre-PNe $(\mathrm{pPNe})$ and PNe. More than $80 \%$ of $\mathrm{PNe}$ are not spherical, but show a variety of 


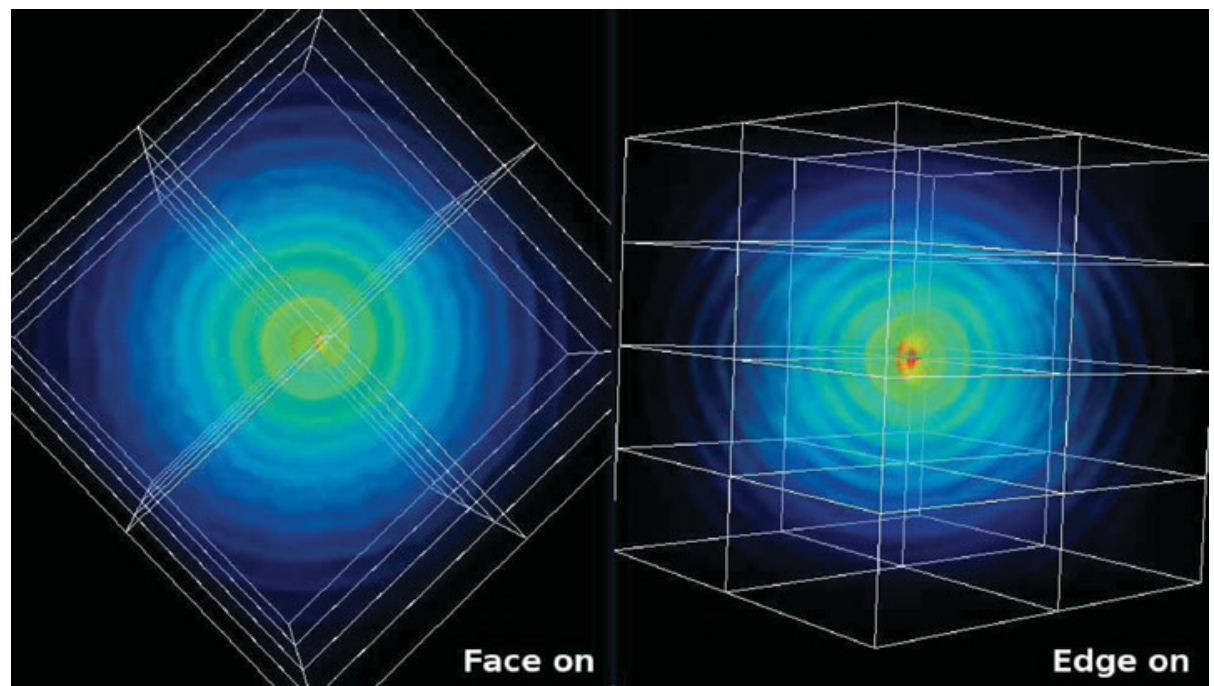

Figure 2. Circumstellar spiral-shell pattern induced by a binary in a circular orbit viewed at (left) face-on and (right) edge-on. The hydrodynamic simulation is performed using the FLASH3.0 code. The density levels vary from blue (low) to red (high). Notice that the edge-on pattern consists of bicentric half-circles. Also notice the overall flattened morphology. Credit: Hsieh, Kim, \& Liu.

morphologies including highly bipolar or multipolar structures, and many of them also have outer faint ring-like patterns. The recurrent patterns have timescales of the order of a few hundred years, which correspond to the periods of wide binary stars. In contrast, the bipolar structures are the expected results of close binary interactions. I propose that a model involving a binary star system in an eccentric orbit can provide a plausible interpretation for the coexistence of these two geometrically distinct structures.

\section{Binary-induced spiral-shell model}

In a binary system, the orbital motion of the mass-losing AGB star introduces an anisotropy in the actual speeds of wind gusts (Soker 1994). The faster and slower wind gusts ejected over the orbit meet each other in the circumstellar space, forming a spiral pattern in the direction following the orbit. Mastrodemos \& Morris (1999) showed, through smoothed-particle hydrodynamics simulations, the three dimensional spiral-shell nature of the circumstellar pattern induced by the binary orbital motion. The detailed structures are described in separate theoretical investigations for the patterns driven by the orbital motion of substellar-mass (e.g., planetary) companions (Kim \& Taam 2012a) and by the reflex motion of a mass-losing star in a comparable-mass binary system (Kim \& Taam 2012b). It is shown that the resulting geometrical details can be used to constrain the stellar masses and orbital properties (Kim \& Taam 2012c; Kim et al. 2013).

The spiral-shell model provides a framework for understanding the various recurrent patterns, which include not only a perfect spiral but also circular rings depending on the orbit inclination angle (face-on vs. edge-on). In the edge-on view, the pattern shape is bicentric half-circles, in general; but when the orbital speed is sufficiently low compared to the wind speed, it takes the form of nearly concentric rings (see e.g., Fig. 2). The projected shape becomes an elongated spiral at intermediate inclination angles, from which the inclination angle can be derived (Fig. 3, and see Kim \& Taam 2012c for details).

Using a simple geometric model, which is completely defined by five equations in Kim \& 

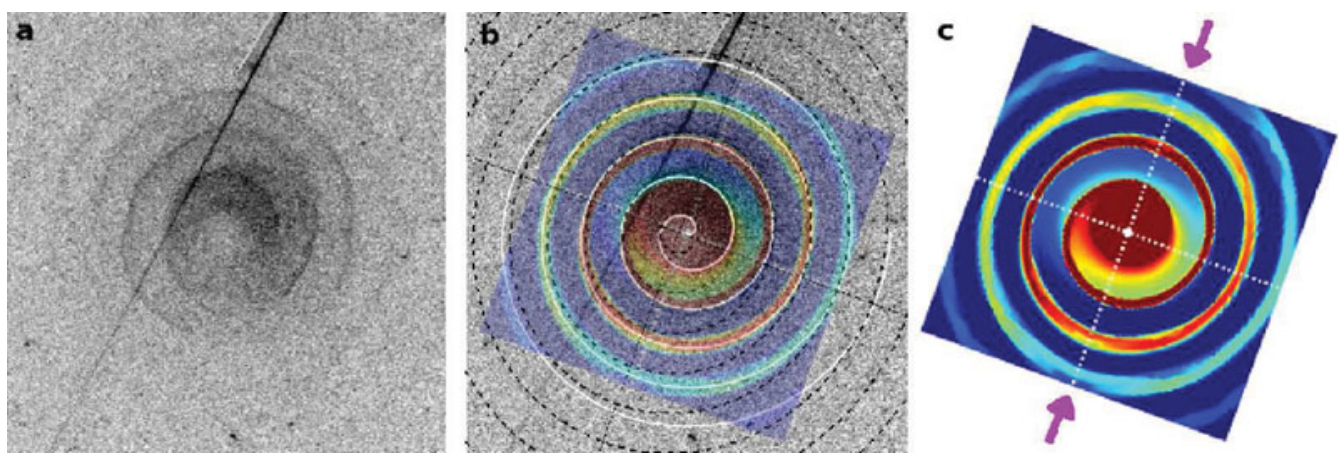

Figure 3. (a-b) AFGL 3068's HST image $\left(25^{\prime \prime} 6 \times 25^{\prime \prime} 6\right)$ is compared with $(\mathrm{b}-\mathrm{c})$ the density distribution in a hydrodynamic model (Kim \& Taam 2012c). The arrows in (c) denotes the line of nodes of the binary orbital plane with the plane of the sky. The orbital period is almost free from the projection effect, and in this case, it is about 800 years.

Taam(2012c), a parameter space analysis for a projected spiral image can be performed. With four observables, mostly measured from the pattern shape, all binary orbital parameters can be derived. Once the parameter space is constrained, hydrodynamic models can be explored to fine tune the binary properties. Because the hydrodynamic modeling is expensive and time consuming, this pre-simulation analysis is very useful.

When an elongated spiral is projected onto a two-dimensional image, it could indicate an oblate ellipsoid halo viewed with a small inclination angle or an almost spherical halo with a very large inclination angle. This degeneracy is inherent in two-dimensional images such as that obtained from the HST. In the current era, high-resolution highsensitivity interferometric data of molecular line emissions can be obtained providing kinematic information and, thus, the three-dimensional distribution of the pattern, lifting the degeneracy (see e.g., Kim et al. 2013 for details).

\section{Eccentric long-period binaries}

ALMA observations now reveal the detailed three-dimensional structure of the unambiguous and complete spiral pattern in AFGL 3068. In particular, the molecular line emission shows the innermost winding of the spiral pattern, which was absent in the HST dust scattered light image. It indicates that the innermost region of the CSE of AFGL 3068 is indeed extremely dense, thus, the penetration of interstellar UV photons required for scattering by the circumstellar dust is prohibited (see Fig. 1, leftmost panels). For the remaining windings, the dust and gas are well coupled.

The most exciting finding from the ALMA map of AFGL 3068 is the bifurcation of the spiral pattern, which is well reproduced by a highly eccentric-orbit binary model and cannot be mimicked by a circular-orbit model with any inclination (Fig. 4; Kim et al. 2017). This is the first solid piece of evidence for the existence of an eccentric binary system from a spiral pattern. We note that it is surprising that even the nearly perfect spiral source is not a circular-orbit binary. The similar and different patterns of various molecular line emissions are subject to detailed study of the radiative transfer and chemical compositional considerations to understand the physical conditions across the spiral shock.

An eccentric binary has also been suggested for another carbon star, CIT 6 , based on the Submillimeter Array (SMA) observation (Fig. 5; see Kim et al. 2015): 1) the onesided gaps of interarm emission, which can be reproduced by an eccentric binary model 


\section{OBSERVATION}

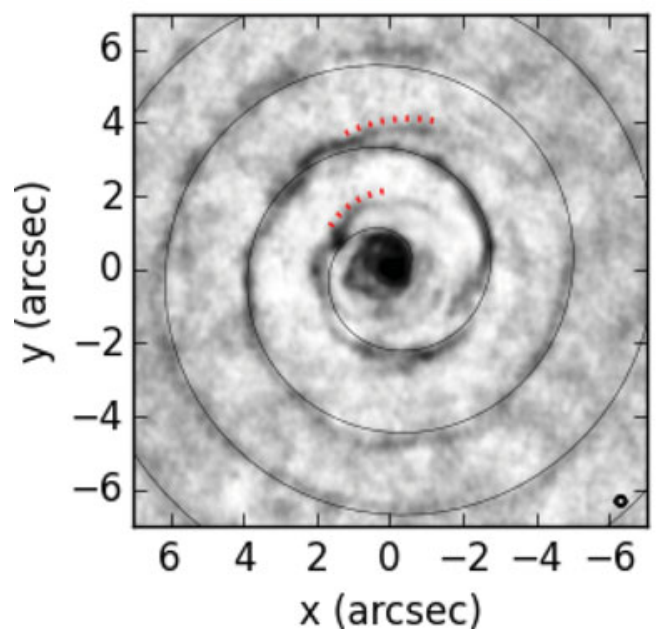

MODEL

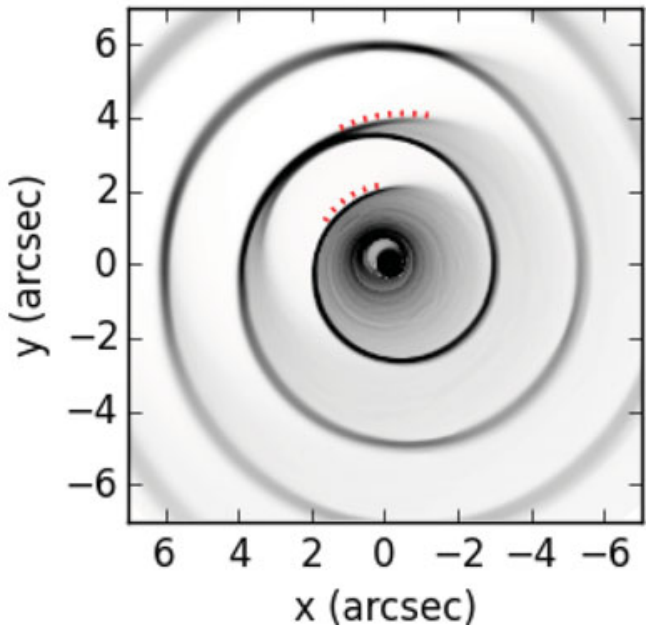

Figure 4. Bifurcation of the spiral pattern in AFGL 3068 revealing the eccentric binary nature. (left) ALMA observation versus (right) a hydrodynamic model of a binary with a large eccentricity of 0.8 . The bifurcation is a distinctive characteristic of an eccentric binary and cannot be reproduced by a circular binary model (Kim et al. 2017).

(e.g. He 2007; Raga et al. 2011); 2) inner double spiral feature, which can be found in a relatively slow wind outflow (e.g., Mohamed \& Podsiadlowski 2012) - the speculation is that a highly eccentric orbit leads to the penetration of the companion into the wind acceleration zone; 3) nascent bipolar (or multipolar) structure at the line edges, which would be a result of strong interaction between the binary stars at periastron passages.

In Table 2, these two carbon stars proposed as members of long-period eccentric binaries are compared to $\mathrm{V}$ Hydrae, a carbon star that is showing bullet-like mass ejection every 8.5 years, which is the possible orbital period of a binary (Sahai et al. 2016). Indeed, the derived orbital periods have a relation with the presence and geometry of the bipolar structures. For a binary in a circular orbit with these long periods, there is no mechanism to produce to the bipolar structure. Perhaps, strong interactions at the close periastron passages for a component in an eccentric binary can lead to a gradual decrease in the orbital separation, eventually leading to the formation of a disk around the companion star where bipolar outflows can be launched. This remains as a hypothesis and more theoretical investigations are needed. If true, the orbital eccentricity must be a key parameter linking the observed spiral-shell patterns to the bipolar structures.

Is it reasonable to expect such high eccentric orbits for these objects? Raghavan et al. (2010) showed that the distribution of orbital eccentricity in main-sequence binaries is random for systems with orbital periods greater than 10 days. Refer to the papers by D. Kamath and H. Van Winckel in this volume for the recent results for the eccentricity distribution of red giant binaries. The above three objects with orbital periods longer than several years are well within the regime that the circularization does not occur.

A triple system is another possible scenario for explaining the coexistence of outer long-period spiral-shell patterns and inner bipolar structures, although it should be very fine-tuned. The primary and secondary objects should be distant to explain the timescale of outer pattern spacing as observed to be of the order of a few hundred years, while the third body should be located near the companion star launching the bipolar structure. Among possible triple system configurations, only one such configuration works to explain the coexistence of two distinguished structures. Since the main-sequence fraction of triple 

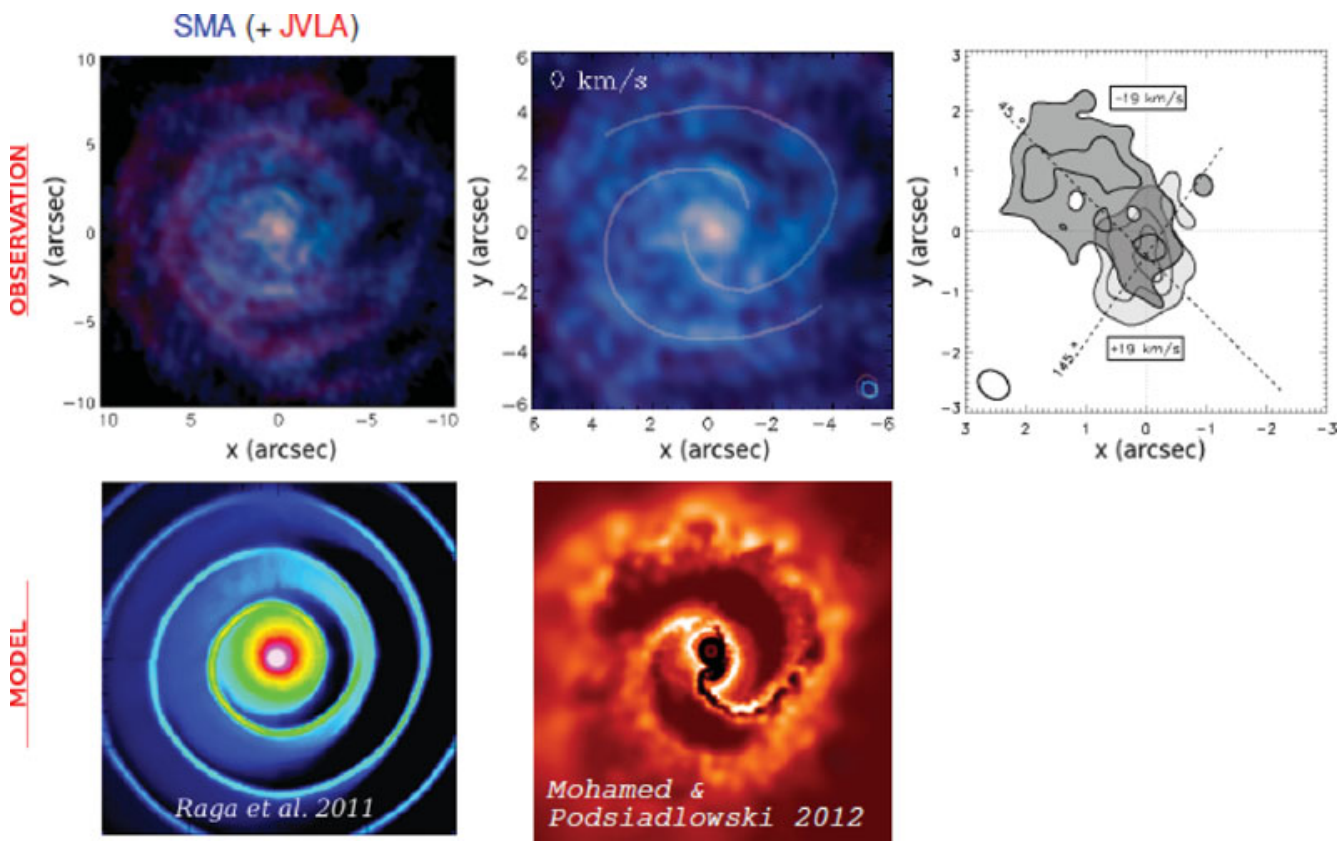

Figure 5. Hints of an eccentric binary in CIT 6 (Kim et al. 2015): (left) one-sided gaps of interarm emission, (middle) inner double spiral feature, and (right) nascent bipolar (or multipolar) structure. These features of CIT 6 revealed in the SMA observations are compared with the hydrodynamic model for a highly eccentric-orbit binary by Raga et al. (2011) and the slow wind model by Mohamed \& Podsiadlowski(2012), respectively.

\begin{tabular}{c|c|c}
\hline AFGL 3068 & CIT 6 & V Hydrae \\
\hline $\begin{array}{c}\text { n 800 years } \\
\text { no bipolar } \\
\text { (Kim et al. } 2017)\end{array}$ & $\begin{array}{c}\sim \text { 300 years } \\
\text { broadened bipolar } \\
\text { (Kim et al. } 2015)\end{array}$ & $\begin{array}{c}8.5 \text { years } \\
\text { bullet-like ejection } \\
\text { (Sahai } \text { et al. } 2016)\end{array}$ \\
\hline
\end{tabular}

Table 2. Comparison of three carbon stars AFGL 3068, CIT6, and V Hydrae tends to show a relation between the presence (and geometry) of bipolar structures and the orbital periods derived from either the spiral pattern or the mass ejection.

and higher-order multiple systems is less than $10 \%$, it would be difficult to understand all known pPNe and PNe having recurrent patterns ( $>60$ objects, according to RamosLarios et al. 2016) by triple systems.

\section{Implications}

Spiral patterns are now frequently detected in the CSEs of stars in the AGB phase thanks to high-sensitivity high-resolution submillimeter/millimeter observations. This is a major step forward as many of them were not obvious in the dust scattered light images (Fig. 1). The complex rose-window pattern of IRC+10216 is understood in terms of a binary system, but requiring a mass loss variation (Cernicharo et al. 2015). For R Scl, a star that has a spiral and a thermal pulse shell, Maercker et al. (2012) used the spiral property as a timer to estimate the thermal pulsation period. In the case of Mira, the ALMA data reveal overall complex structures, but because it has a confirmed white dwarf companion, the partial arcs could be connected as a spiral (Ramstedt et al. 2014). 
Many of these objects have a secondary point-like source as revealed in the near-infrared, optical, or radio continuum with apparent projected separations greater than $50 \mathrm{AU}$.

These five, best-known spiral/shell/arc objects are all in the AGB phase and are mostly carbon-rich. In order to determine whether the presence and evolution of circumstellar patterns depend on the stellar evolutionary phase and the chemical environment, molecular line surveys for AGB spirals in post-AGB sources are needed. Given such surveys, we can contemplate carrying out statistical studies of the known spiral/ring/arc sources. At the same time, analysis of the simplest objects, coupled with theoretical studies, will enable parameter estimation for a statistically meaningful sample. The golden era for studying the spiral-shell patterns is here, allowing us to progress in unravelling the nature and evolution of these evolved stars as encoded in their circumstellar patterns.

\section{Acknowledgements}

H.K. acknowlenges support for the travel and the work presented in this paper through EACOA Fellowship from the East Asia Core Observatories Association, which consists of the National Astronomical Observatories of China, the National Astronomical Observatory of Japan, the Korea Astronomy and Space Science Institute, and the Academia Sinica Institute of Astronomy and Astrophysics.

\section{References}

Cernicharo, J., Marcelino, N., Agúndez, M., \& Guélin, M. 2015, A\&A, 575, A91

Corradi, R. L. M., Sánchez-Blázquez, P., Mellema, G., Giammanco, C., \& Schwarz, H. E. 2004, A\&A, 417, 637

He, J. H. 2007, A\&A, 467, 1081

Kim, H. \& Taam, R. E. 2012a, ApJ, 744, 136

Kim, H. \& Taam, R. E. 2012b, ApJ, 759, 59

Kim, H. \& Taam, R. E. 2012c, ApJL, 759, L22

Kim, H., Hsieh, I.-T., Liu, S.-Y., \& Taam, R. E. 2013, ApJ, 776, 86

Kim, H., Liu, S.-Y., Hirano, N., et al. 2015, ApJ, 814, 61

Kim, H., Trejo, A., Liu, S.-Y., et al. 2017, Nat. Astron., 1, 0060

Leão, I. C., de Laverny, P., Mékarnia, D., de Medeiros, J. R., \& Vandame, B. 2006, A\&A, 455, 187

Maercker, M., Mohamed, S., Vlemmings, W. H. T., et al. 2012, Nature, 490, 232

Mastrodemos, N. \& Morris, M. 1999, ApJ, 523, 357

Meijerink, R., Mellema, G., \& Simis, Y. 2003, A\&A, 405, 1075

Mohamed, S. \& Podsiadlowski, P. 2012, Baltic Astronomy, 21, 88

Olofsson, H., Maercker, M., Eriksson, K., Gustafsson, B., \& Schöier, F. 2010, A\&A, 515, A27

Raga, A. C., Cantó, J., Esquivel, A., Huggins, P. J., \& Mauron, N. 2011, in Asymmetric Planetary Nebulae V Conference, ed. A. A. Zijlstra et al. (Jodrell Bank Centre for Astrophysics, Manchester), 185

Raghavan, D., McAlister, H. A., Henry, T. J., et al. 2010, ApJS, 190, 1

Ramos-Larios, G., Santamaría, E., Guerrero, M. A., et al. 2016, MNRAS, 462, 610

Ramstedt, S., Mohamed, S., Vlemmings, W. H. T., et al. 2014, A\&A, 570, L14

Sahai, R., Trauger, J. T., Watson, A. M., et al. 1998, ApJ, 493, 301

Sahai, R., Scibelli, S., \& Morris, M. R. 2016, ApJ, 827, 92

Schmidt, G. D., Hines, D. C., \& Swift, S. 2002, ApJ, 576, 429

Soker, N. 1994, MNRAS, 270, 774

Su, K. Y. L. 2004, in ASP Conf. Ser. 313, Asymmetrical Planetary Nebulae III: Winds, Structure and the Thunderbird, ed. M. Meixner et al. (San Francisco, CA: ASP), 247 


\section{Discussion}

CORRADI: Statistically, you should observe more circular and inclined spiral pattern rather than the spiral pattern that you expect when the systems are observed from "the pole". Is it what you see? Also, in PNe most patterns are clearly circular (e.g. NGC 6543), while in AGB stars you are mainly finding spirals.

KIM: The rings in PNe could be any of spirals or rings. Get a spiral model, block the central part by your hand, then you will find you cannot distinguish spiral/ring any more. Try it at home. Statistically, spirals are major in the binary model at the inclination angles $0<=\mathrm{i}<=70 \mathrm{deg}$. except for the very edge-on case.

TORRES-PEIMBERT: Do you have any restrictions about the mass loss rate in your models?

KIM: The mass loss rate is used as an input parameter of the hydro dynamic model. With the full intensity data and through a radiative transfer calculation of the hydro dynamic model, the total mass distribution over the circumstellar envelope, and thus the rate, can be obtained. The constraint for the mass loss rate is possibly different for single vs. binary models, and for different inclination angles of the binary orbit.

De Marco: Have you determined what type of companion is implied by the ring structure of the Cat's Eye nebula? This PN is so close that we have put stringent limits on the companion mass and location ...

KIM: Not Yet. Let's do that.

LIU: It seems that observations hitherto of extrasolar planetary systems (proto-stellar disks) reveal spiral arms only in reflection light, not in e.g. dust emission. The case seems to be different in evolved stars (AGBs) or PNe. Would you like to comment on this?

Muthumariappan: Can one say something on the masses of and mass-loss rates from these spirals?

KwoK: You explain the outer structure as 2-dimensional spirals, but do you know that they are not projected 3-D shells? 